\title{
Remoción de azul brillante mediante columnas empacadas usando tuza de maíz
}

\section{Removal of the brilliant blue through packed columns using corncobs}

\author{
Remoção de azul brilhante, utilizando colunas tuza \\ milho embalados
}

\author{
Angelina Hormaza Anaguano ${ }^{1}$, Yurany Andrea Villada Villada² \& Laura Mariana Orrego Roldán ${ }^{3}$ \\ 'Químico, Magister en Ciencias -Química Orgánica, Doctor Rerum Naturalien, PhD en Ciencias \\ Químicas. ${ }^{2}$ Ingeniera Química, Joven investigador. ${ }^{3}$ Estudiante de Ingeniería Química \\ ${ }^{1,2}{ }^{2}$ Escuela de Química, Facultad de Ciencias, ${ }^{3}$ Escuela de Procesos y Energía, Facultad de Minas. \\ 1,2,3Universidad Nacional de Colombia, Sede Medellín.

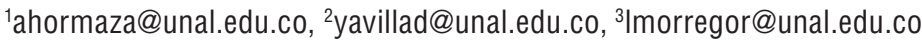

\section{Resumen}

Esta investigación muestra el potencial adsorbente de la tuza de maíz en la remoción del colorante aniónico azul brillante sobre lechos empacados. Se evaluó efecto de los parámetros $\mathrm{pH}$ del medio, tamaño de partícula, presencia salina y altura de columna. Las muestras fueron analizadas a intervalos definidos de tiempo. La cantidad del colorante removido fue cuantificada por medio de espectroscopia Ultravioleta - Visible. Los modelos de Adams-Bohart, Thomas y Yoon-Nelson fueron utilizados para predecir las curvas de ruptura usando regresión no lineal y con ello establecer los parámetros característicos del proceso. El modelo BDST fue empleado para expresar la influencia de la altura del lecho en la curva de ruptura. Se encontró que a $\mathrm{pH}$ ácido $(\mathrm{pH}=2.0)$ menor tamaño de partícula $(0.3 \mathrm{~mm}<\mathrm{x}<0.5 \mathrm{~mm})$ y mayor altura de lecho $(Z=20 \mathrm{~cm})$ se favorece la transferencia de colorante al material adsorbente, alcanzando mayor retención del azul brillante sobre la tuza de maíz. La presencia de $\mathrm{NaCl}$ en la solución reduce la eficiencia en la adsorción. En cuanto a modelos, el de Thomas presentó el mejor ajuste para la descripción de las curvas de ruptura a las condiciones experimentales $\left(R^{2}=0.93-0.94\right)$, mientras Adams-Bohart ofreció un buen ajuste $\left(R^{2}=0.96\right.$ -
0.99) para el comportamiento dinámico limitado a la parte inicial del proceso. Yoon-Nelson presentó un desvío considerable entre la información experimental y la predicha. Finalmente, el BDST mostró una adecuada correlación, $\mathrm{R}^{2}=0.92-0.99$, estableciendo que la altura es determinante para el escalado del proceso.

Palabras clave: adsorción en continuo, altura de lecho, colorante aniónico, curva de ruptura, efecto salino.

\section{Abstract}

This research shows the adsorbent potential of corncobs in the removal of the anionic bright blue colorant on packed beds. It was evaluated the effect of $\mathrm{pH}$ parameters from this medium, particle size, saline presence and column height. The samples were analyzed by defined intervals of time. The colorant quantity removed was quantified by means of ultraviolet-visible spectroscopy. Models of Adams-Bohart, Thomas and Yoon-Nelson were used to predict the breakthrough curves using nonlinear regression and so set the characteristic parameters of the process. BDST model was used to express the influence of bed height into 
the breakthrough curve. It was found that acid $\mathrm{pH}$ $(\mathrm{pH}=2.0)$ smaller particle size $(0.3 \mathrm{~mm}<\mathrm{x}<0.5$ $\mathrm{mm})$ and larger bed height $(Z=20 \mathrm{~cm})$ favor the transfer of colorant to the adsorbent material, reaching greater retention of bright blue on the corncob. The presence of $\mathrm{NaCl}$ in the solution reduces the efficiency of adsorption. In terms of models, the Thomas model presented the best setting for the description of the breakthrough curve to the experimental conditions ( $R 2=0.93-0.94)$, while Adams-Bohart model offered a good fit $(\mathrm{R} 2=0.96$ -0.99) for the dynamic behavior limited to the initial part of the process. Yoon-Nelson model presented a considerable drift between the experimental data and the predicted data. Finally, the BDST model showed a good correlation, R2 = $0.92-0.99$, establishing that the height is a determining factor for scaling of process.

Key-words: adsorption on continuous, bed height, anionic dye, curve of rupture, saline effect

\section{Resumo}

Esta pesquisa mostra o potencial adsorvente do sabugo de milho na remoção do corante aniônico azul brilhante sobre leitos empacotados. Foi avaliado o efeito dos parâmetros $\mathrm{pH}$ do meio, tamanho de partícula, presença de salinidades e altura da coluna. As amostras foram analisadas em interva- los de tempo definidos. A quantidade de corante removido foi quantificada por espectroscopia de UV - Visível. Modelos Adams-Bohart, Thomas e Yoon-Nelson foram utilizados para prever as curvas de ruptura através de regressão não-linear e, assim, estabelecer os parâmetros característicos do processo. O modelo BDST foi utilizado para expressar a influência da altura do leito na curva de ruptura. Verificou-se que a $\mathrm{pH}$ ácido $(\mathrm{pH}=2,0)$ o tamanho de partícula mais pequeno $(0.3 \mathrm{~mm}$ $<x<0,5 \mathrm{~mm})$ e maior altura do leito $(Z=20 \mathrm{~cm})$ favorece a transferência do material adsorvente de corante atingindo uma maior retenção de azul milho brilhante no sabugo. A presença de cloreto de sódio na solução reduz a eficiência de adsorção. Quanto aos modelos, o Thomas apresentou o melhor ajuste para descrever as curvas de ruptura para as condições experimentais $\left(R^{2}=0,93\right.$ a $0,94)$, enquanto Adams-Bohart oferecido um bom ajuste $\left(R^{2}=0,96-0,99\right)$ para o comportamento dinâmico limitado para a parte inicial do processo. Yoon-Nelson apresentou um desvio significativo entre o experimental ao previsto. Por fim, BDST mostrou boa correlação, $R^{2}=0,92-0,99$, estabelecendo que a altura seja crucial para o processo de escala.

Palavras-chave: adsorção contínua, altura do leito, corantes aniônicos, curva de ruptura, efeito salino.

\section{Introducción}

El calentamiento global con el subsecuente cambio climático ha ocasionado en los últimos años un gran número de catástrofes a nivel mundial, impactando tanto las condiciones de vida de la población como sus sectores productivos. Estas devastaciones han ratificado el problema de contaminación de nuestro planeta y la demanda de políticas que permitan contrarrestarlas (El-Fadel, Findikakis \& Leckie, 1997).

La implementación de tecnologías limpias para los diferentes procesos industriales constituye una de las soluciones más certeras a esta problemática. Entendiéndose por tecnología limpia todo aquel proceso que propende por un mínimo o nulo impacto ambiental y que aprovecha de forma eficiente materiales de desecho y materiales avanzados (Pardo, 2012).

En este sentido, la utilización de adsorbentes alternativos, tal como los subproductos agrícolas, representa un aporte valioso en el delineamiento de estrategias eficientes, de bajo costo y ambientalmente favorables para el tratamiento de efluentes 
coloreados (Rafatullah, 2010; Gupta \& Suhas 2009; Crini, 2006). La amplia disponibilidad de estos residuos agrícolas conlleva a una notable reducción de costos en la implementación del proceso de adsorción y reemplaza el uso tradicional de carbón activado, que pese a su eficiencia en la retención de múltiples contaminantes disueltos, tiene como principales limitantes su alto precio de adquisición y la dificultad de su regeneración (Wang, Zhu Coomes, Haghseresht \& Lu, 2005).

El azul brillante, $A B$, es un colorante aniónico usado ampliamente en la industria textil, de cueros y en la industria alimentaria como aditivo en bebidas, dulces, extractos y condimentos (Mittal, 2005). En humanos es bastante tóxico ocasionando desórdenes carcinogénicos, reproductivos y neurológicos (Gupta, Mittal, Krihsnan \& Mittal J., 2005). Además, a nivel del hábitat, el ecosistema acuático se ve afectado con su presencia, no sólo por el deterioro estético, sino ante todo por la reducción del paso de la radiación solar incidente, inhibiendo de esta forma el proceso de fotosíntesis (Asku \& Isoglu, 2006).

Los residuos agroindustriales han sido ampliamente explorados como adsorbentes potenciales en la remoción de colorantes disueltos (Song, Zou, Bian, Su \& Han, 2011) debido a sus propiedades superficiales y facilidad de obtención, mostrando una alta capacidad para la retención de estos contaminantes. En particular, la tuza de maíz, constituida principalmente por celulosa y hemicelulosa, ha mostrado una alta capacidad de adsorción tanto de colorantes como de metales pesados (Buasri, Chaiyut, Tapang, Jaroensin \& Panphtom, 2012; Hormaza, Figueroa \& Moreno, 2012; Moreno, Hormaza \& Figueroa, 2012), convirtiéndose así en una excelente opción al momento de elegir un buen adsorbente.

Es valioso mencionar que se ha reportado la remoción de $A B$ sobre diversos materiales de desecho, tales como residuos de flores (Jaramillo, Echavarria, \& Hormaza, 2013), cenizas de combustión y soya (Gupta, Mittal, Krishnan \& Mittal, J., 2005), arcillas
(Ketelsen \& Meyer-Windel, 1999) y banano (Namasivayam, Prabha \& Kumutha, 1998) pero limitado al proceso bajo sistema discontinuo. Esta metodología ofrece sin lugar a duda información valiosa sobre la eficiencia del sistema adsorbente-adsorbato. No obstante, es insuficiente a la hora de llevar a cabo su escalado. Por tanto, es claro que para la magnificación del proceso se precisa de su evaluación bajo sistema continuo, como metodología más cercana a un tratamiento de interés industrial que implica el manejo de grandes volúmenes de efluentes coloreados. (Han, Wang, Yu, Zou, Shi \& Liu, 2007; Han, Ding, Xu, Zou, Wang, Li \& Zou, 2008; Han, Wang, Zhao, Wang, Xie, Cheng \& Tang, 2009).

En concordancia con lo anterior, el propósito de la presente investigación fue explorar la posibilidad de utilizar la tuza de maíz para la remoción del AB mediante lechos empacados. Así, el objetivo de este trabajo fue llevar a cabo la evaluación de las principales variables, $\mathrm{pH}$ de la solución, tamaño de partícula, presencia de $\mathrm{NaCl}$ y altura del lecho en la remoción en continuo del colorante AB. La presencia de $\mathrm{NaCl}$ se evalúo con el propósito de simular condiciones más cercanas a las realmente exhibidas por los efluentes coloreados procedentes de la industria textil, dado que en el proceso de tinción, esta sal es ampliamente utilizada para la fijación del color (Han, Wang, Yu, Zou, Shi, \& Liu, 2007; Han, Ding, Xu, Zou, Wang, Li, \& Zou, 2008). Para determinar la completa información del proceso, se utilizaron los modelos de Adams-Bohart, Thomas, Yoon-Nelson y depth/service time analyse (BDST) como bases para la estimación de los parámetros característicos de la adsorción.

\section{Modelado en columnas}

El rendimiento de una columna de lecho fijo se describe a través del concepto de la curva de ruptura. El tiempo de ruptura y la forma de la curva son características muy importantes para la determinación de la operación y respuesta dinámica de una columna de adsorción (Han et al., 2009). 


\section{Modelo de Thomas}

El modelo de Thomas es uno de los métodos más usados en la representación y análisis de datos obtenidos a partir de ensayos en columnas. Generalmente se utiliza para conocer la máxima capacidad de adsorción del material adsorbente y la constante cinética. (Han et al., 2007). La expresión del modelo de Thomas para la adsorción en columnas se representa a través de la ecuación (1).

$$
\frac{C_{t}}{C_{0}}=\frac{1}{1+\exp \left(k_{T h} q_{0} x / v-k_{T h} C_{0} t\right)}
$$

Donde $\mathrm{K}_{\mathrm{Th}}$ es la constante de Thomas $\left(\mathrm{mL} \mathrm{min}^{-1}\right.$ $\left.\mathrm{mg}^{-1}\right) ; \mathrm{q}_{0}$ es la captación de AB por gramo de adsorbente ( $\mathrm{mg} \mathrm{g} \mathrm{g}^{-1}$ ); $x$ es la cantidad de adsorbente en la columna (g); $\mathrm{C}_{0}$ es la concentración inicial del influente de $A B\left(\mathrm{mgL}^{-1}\right) ; \mathrm{C}_{t}$ es la concentración del efluente en el tiempo $t\left(\mathrm{mgL}^{-1}\right)$ y $v$ es el flujo de entrada $\left(\mathrm{mL} \min ^{-1}\right)$.

\section{Modelo de Adams-Bohart}

El modelo de Adams-Bohart describe la parte inicial de las curvas de ruptura, se usa para la estimación de algunos parámetros como la capacidad de adsorción $\left(\mathrm{N}_{0}\right)$ y la constante cinética $\left(\mathrm{k}_{\mathrm{AB}}\right)$, (Han et al., 2007). La expresión de Adams-Bohart para el estudio en columnas es descrita mediante la ecuación (2).

$$
\frac{C_{t}}{C_{0}}=\exp \left(k_{A B} C_{0} t-k_{A B} N_{0} \frac{z}{F}\right)
$$

Donde $\mathrm{K}_{\mathrm{AB}}$ es la constante cinética $\left(\mathrm{L} \mathrm{mg}^{-1} \mathrm{~min}^{-1}\right), \mathrm{F}$ es la velocidad lineal calculada dividiendo el flujo por el área transversal de la columna (cm min-1), $Z$ es la altura del lecho en la columna y $\mathrm{N}_{0}$ es la concentración de saturación (mg L-1).

\section{Modelo de Yoon- Nelson}

El modelo de Yoon-Nelson para analizar el comportamiento en la curva de ruptura, de un sistema de un solo componente, está dado por la ecuación (3).

$$
\frac{c_{t}}{c_{o}-c_{t}}=\exp \left(k_{Y N} t-\tau k_{Y N}\right)
$$

Donde $\boldsymbol{k}_{\boldsymbol{Y N}}$ es la constante de velocidad $\left(\mathrm{min}^{-1}\right)$ y $\tau$ es el tiempo requerido para alcanzar el $50 \%$ de AM adsorbido en la curva. Los parámetros del modelo pueden ser obtenidos usando el método de regresión no-lineal.

\section{Modelo BDST}

El modelo se basa en la medición de la capacidad adsortiva del lecho en diferentes valores de la curva de avance. Proporciona además las ecuaciones de modelado útiles para los cambios de parámetros del sistema. La ecuación (4) expresa el tiempo en la curva de avance como función de los parámetros de operación en el modelo BDST (Han et al., 2007).

$$
t=\frac{N_{O}}{c_{0}} Z-\frac{1}{k_{a} c_{a}} \ln \left(\frac{c_{0}}{c_{T}}-1\right)
$$

Donde $C_{t}$ es la concentración de la solución en el tiempo $\left(\mathrm{mg} \mathrm{L}^{-1}\right) \mathrm{C}_{0}$ la concentración inicial de la solución ( $\left.\mathrm{mg} \mathrm{L}^{-1}\right)$, $\mathrm{F}$ el flujo de entrada $\left(\mathrm{cm} \mathrm{min}^{-1}\right)$, $\mathrm{N}_{0}$ la capacidad de adsorción ( $\left.\mathrm{mg} \mathrm{g}^{-1}\right), \mathrm{K}_{\mathrm{a}}$ la constante cinética $\left(\mathrm{L} \mathrm{mg}^{-1} \mathrm{~min}^{-1}\right)$, $\mathrm{t}$ el tiempo (min) y $\mathrm{Z}$ es la altura de lecho en la columna $(\mathrm{cm})$, (Han et al., 2007). 


\section{Análisis de error}

La regresión no lineal es una función de tipo $\boldsymbol{y}=\boldsymbol{f}(\boldsymbol{x}, \boldsymbol{\theta})+\boldsymbol{\varepsilon}$ basada en datos multidime sionales $\boldsymbol{x}, \boldsymbol{y}$, donde $\boldsymbol{f}$ es una función no lineal respecto a algunos parámetros desconocidos $\theta$, que permite obtener los valores de los parámetros asociados a la mejor curva de ajuste a través del método de los mínimos cuadrados. Para el presente estudio, se realiza un análisis estadístico con el coeficiente de correlación $\left(R^{2}\right.$, ecuación 5$)$ y el error estadístico (SS, ecuación 6) para calcular la probabilidad de error.

$$
\begin{array}{r}
R^{2}=\frac{\sum_{i=1}^{n}\left(\widehat{y}_{i}-\bar{y}\right)^{2}}{\sum_{i=1}^{n}\left(y_{i}-\bar{y}\right)^{2}} \\
S S=\sqrt{\sum \frac{\left(y_{B}-y_{c}\right)^{2}}{N}}
\end{array}
$$

De la ecuación 6 , ye y yc corresponden al valor experimental y calculado según el modelo y $\mathrm{N}$ es el número de experimentos (Han et al., 2007).

\section{Materiales y métodos}

\section{Preparación del adsorbente}

La tuza de maíz, TM, se recolectó en la Plaza Minorista de la ciudad de Medellín, se sometió a un proceso de molienda y tamizado para clasificar el material según el tamaño de partícula deseado. Posteriormente se lavó en repetidas ocasiones con agua destilada y peróxido de hidrógeno (5\%) con el fin de obtener el material libre de materia orgánica y luego, se volvió a someter a secado a $120^{\circ} \mathrm{C}$ durante 24 horas. El material adsorbente con tamaño de partícula entre $0.3-0.7 \mathrm{~mm}$ fue conservado en recipientes herméticos de vidirio para los ensayos posteriores.

\section{Preparación de la solución de azul brillante}

La solución de $\mathrm{AB}$ se preparó en agua destilada a una concentración de $100 \mathrm{~m} \mathrm{~L}^{-1}$ ajustando el valor de $\mathrm{pH}$ deseado con alícuotas de soluciones de $\mathrm{HCl} e$ $\mathrm{NaOH} 0.1 \mathrm{M}$. Para el estudio de la influencia del $\mathrm{NaCl}$ en el proceso de adsorción, se preparó la solución a las mismas condiciones, pero adicionando además $100 \mathrm{~g}$ de $\mathrm{NaCl}$ por cada gramo de colorante, dicha relación $\mathrm{NaCl} /$ colorante se aproxima a la empleada en la industria textil para el proceso de tinción.

\section{Estudio de adsorción en continuo}

Los experimentos de adsorción a flujo continuo fueron realizados en una columna de vidrio, cuyas dimensiones generales fueron $2.6 \mathrm{~cm}$ de diámetro interno y $48 \mathrm{~cm}$ de altura. La columna opera en flujo ascendente, controlado con una bomba peristáltica marca VWR flujo medio-alto. Las muestras para la medición y análisis de resultados, se colectaron a la salida de la columna a intervalos regulares de tiempo hasta alcanzar la saturación total del lecho. La concentración de $A B$ en cada una de las muestras recolectadas fue cuantificada por medio de espectroscopia de UV-Vis a una longitud de onda máxima de $\lambda_{\text {máx }}=690 \mathrm{~nm}$, obtenida a partir de un análisis experimental (Jaramillo, Echavarria \& Hormaza, 2013; Auxilio et al., 2007; Khataee \& Khataee, 2008).

Las variables evaluadas fueron, $\mathrm{pH}$ de la solución, tamaño de partícula del adsorbente y altura del lecho empacado. Los intervalos seleccionados fueron: $\mathrm{pH}$ del medio: 2.0, 6.0 y 9.0; tamaño de partícula: $0.3 \mathrm{~mm}<x<0.5 \mathrm{~mm}$ y $0.5 \mathrm{~mm}<x<0.7$ $\mathrm{mm}$, y altura del lecho: $Z=10,15$ y $20 \mathrm{~cm}$, dejando constante la velocidad de entrada $\left(\mathrm{V}=20 \mathrm{~mL} \mathrm{~min}^{-1}\right)$ y la concentración del colorante $\left(\mathrm{C}_{0}=100 \mathrm{mg} \mathrm{L}^{-1}\right)$. Su efecto fue analizado tomando como base las curvas de ruptura resultantes de cada experimento, en donde se evalúo un determinado parámetro y se mantuvieron fijos los restantes con el fin de apreciar claramente su influencia. 
Adicionalmente, el efecto de la presencia salina, $\mathrm{NaCl}$, en la adsorción de AB sobre TM fue evaluado comparando los resultados obtenidos en las curvas de ruptura en ausencia y presencia de $\mathrm{NaCl}$, con una altura de lecho de $10 \mathrm{~cm}$, a una concentración inicial de $100 \mathrm{mg} \mathrm{L}^{-1}$ y flujo de entrada constante de 20 $\mathrm{mL} \mathrm{min}^{-1}$. La cantidad de $\mathrm{NaCl}$ utilizada corresponde a la empleada en la industria textil para la tinción del material, es decir, $100 \mathrm{~g}$ de $\mathrm{NaCl}$ por cada gramo de colorante. Cabe señalar que sales como el $\mathrm{CaCl}_{2}$ pueden contribuir en mayor medida a la generación de cargas positivas sobre la superficie del adsorbente en comparación con el $\mathrm{NaCl}$. No obstante, el mínimo costo de esta última, la ha convertido en la sal seleccionada para el proceso de adsorción.

\section{Estimación de las curvas de ruptura}

Para describir el comportamiento y algunas aplicaciones a escala industrial del proceso, los resultados experimentales se ajustaron a los modelos de Thomas, Adams-Bohart, Yoon-Nelson y BDST, analizando como variable principal el efecto del tamaño del lecho, mediante un análisis de regresión no lineal, para obtener las características principales y determinar las mejores condiciones de operación del sistema. Así mismo se utilizó el error estadístico SS y el factor de correlación $\mathrm{R}^{2}$ como verificadores del modelo usado. Para el procesamiento de los datos se utilizó el programa de Microsoft Office Excel y el complemento de optimización Solver.

\section{Resultados y discusión}

\section{Efecto del pH de la solución inicial en la curva de ruptura}

El efecto del valor del pH en la solución inicial en la adsorción del $A B$ sobre TM se evaluó a los siguientes valores de $\mathrm{pH}=2.0,6.0$ y 9.0. Como se aprecia en la Figura 1, un incremento de $\mathrm{pH}$ desplaza la curva de ruptura de derecha a izquierda, indicando que una cantidad menor de $A B$ es removido y que un período de tiempo menor es requerido para alcanzar la saturación del lecho, reduciendo la eficiencia del proceso de adsorción. Lo anterior permite sugerir que el medio ácido favorece la remoción de este colorante, por esta razón un $\mathrm{pH}=2.0$ fue seleccionado para los siguientes experimentos.

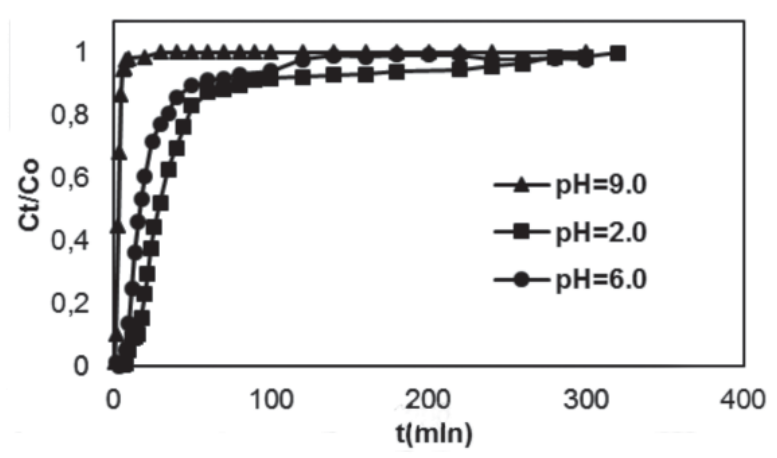

Figura 1. Curvas de ruptura. Análisis del efecto del pH inicial de la solución en la adsorción de $A B-T M$ $\left(C_{0}=100 \mathrm{mg} \mathrm{L}^{-1}, V=20 \mathrm{~mL} \mathrm{~min}{ }^{-1}, Z=10 \mathrm{~cm}\right.$ )

El principal argumento que explica el aumento en la remoción del $A B$ a medida que disminuye el valor del $\mathrm{pH}$ del medio, es el incremento de las fuerzas electrostáticas entre el colorante aniónico $A B$ y los sitios activos cargados positivamente sobre la superficie del material adsorbente, el cual, debido a la adición de un ácido se encuentra protonado. Es conocido que la variación en el valor de $\mathrm{pH}$ de la solución puede afectar significativamente la carga superficial del material y el grado de ionización y especiación del adsorbato (Özcan, Erdem\& Özcan, 2004). El residuo agrícola TM está constituido por un elevado porcentaje de celulosa y hemicelulosa, por tanto, sus grupos funcionales hidroxilos experimentan una protonación como resultado de la adición de un ácido, tal como se ha reportado para otros materiales lignocelulósicos (Piccin, Vieira, Goncalves, Dotto \& Pinto, 2009).

\section{Influencia del tamaño de partícula en la curva de ruptura}

Para determinar la influencia del tamaño de partícula del adsorbente $\mathrm{TM}$, se seleccionaron dos tamaños, $(0.3 \mathrm{~mm}<\mathrm{x}<0.5 \mathrm{~mm})$ y $(0.5 \mathrm{~mm}<\mathrm{x}<0.7$ $\mathrm{mm}$ ) respectivamente. Como se puede apreciar en la Figura 2, un aumento en el tamaño de la partícula desplaza la curva de ruptura de derecha a izquierda indicando que una menor cantidad del colorante 
AB es removido. Así, el proceso se ve favorecido a un tamaño de partícula menor, ya que existe una mayor área superficial expuesta del adsorbente, favoreciendo el área de contacto entre el adsorbente y el adsorbato.

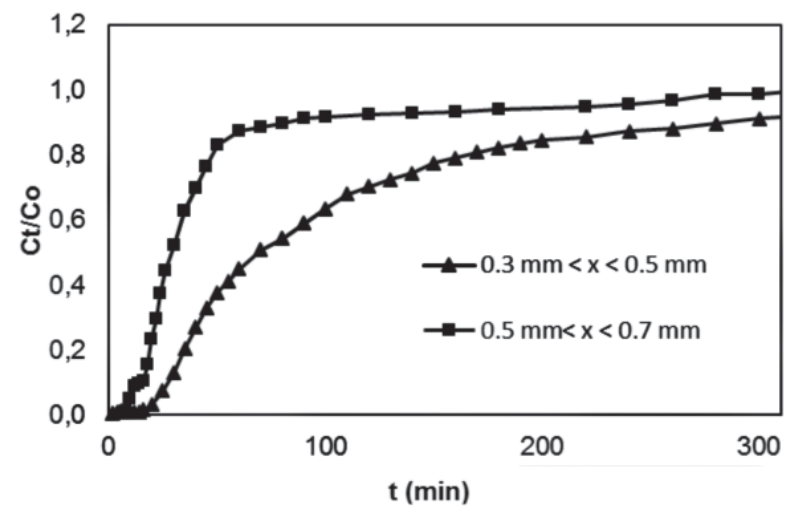

Figura 2. Curvas de ruptura. Análisis del tamaño de partícula en la adsorción de $A B-T M\left(C_{0}=100\right.$ $m g L^{-1}, V=20 \mathrm{~mL} \mathrm{~min}^{-1}, Z=10 \mathrm{~cm}, \mathrm{pH}=2.0$ )

Al comparar por ejemplo el tiempo necesario para alcanzar la saturación de la columna, se observa que con un menor tamaño de partícula se requiere de 190 min, en tanto que con un tamaño de partícula mayor, este tiempo se reduce a $90 \mathrm{~min}$, provocando la rápida saturación del lecho y la imposibilidad de remoción de una mayor cantidad del colorante, que finalmente conducen a una disminución en la eficiencia. Por tanto, un tamaño de partícula entre 0.3 $\mathrm{mm}<\mathrm{x}<0.5 \mathrm{~mm}$ permite tratar un mayor volumen de solución del efluente contaminado.

\section{Influencia de la solución de $\mathrm{NaCl}$}

El comportamiento de las curvas de ruptura de la adsorción de $A B$ sobre TM, en presencia y ausencia de $\mathrm{NaCl}$, es mostrado en la Figura 3. Se observa que la presencia de $\mathrm{NaCl}$ desplaza la curva de derecha a izquierda, indicando una menor retención del colorante AB debido al menor tiempo requerido para alcanzar la saturación, lo cual conduce a una disminución en la eficiencia en proceso.

Así, al comprar las curvas de ruptura, se encuentra que a un tiempo de $45 \mathrm{~min}$ la presencia de $\mathrm{NaCl}$ genera una saturación del lecho del $80 \%$, en tanto que solamente se registra un $60 \%$ de saturación en la columna cuando no existe solución salina, confirmando que la retención del colorante de estudio es más favorable en ausencia de $\mathrm{NaCl}$. Sin embargo, en busca de simular condiciones más cercanas a los efluentes coloreados reales, los ensayos posteriores serán realizados considerado la presencia de $\mathrm{NaCl}$.

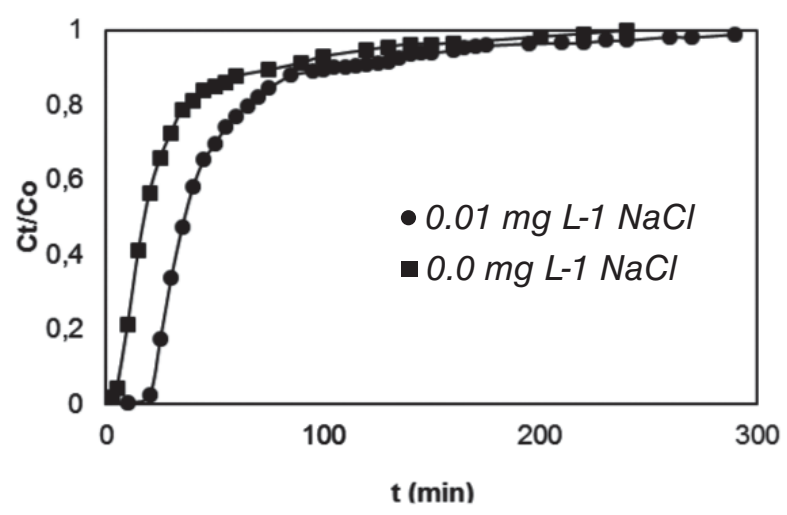

Figura 3. Curvas de ruptura. Análisis del efecto del $\mathrm{NaCl}$ en la adsorción de $A B-T M\left(C_{0}=100 \mathrm{mg} \mathrm{L}^{-1}\right.$, $\left.V=20 \mathrm{~mL} \mathrm{~min}^{-1}, \mathrm{Z}=10 \mathrm{~cm}, \mathrm{pH}=2.0\right)$

La menor retención del $A B$ debido a la presencia de $\mathrm{NaCl}$ puede ser atribuida al efecto competitivo entre los iones del colorante y los aniones cloruro por los sitios activos cargados positivamente presentes sobre la superficie del adsorbente. Los aniones salinos al ocupar dichos sitios reducen la posibilidad de interacción entre el $A B$ y el adsorbente.

\section{Efecto de la altura de la columna}

La influencia de la variable altura (10, 15 y 20 $\mathrm{cm}$ ) sobre las curvas de ruptura se presenta en la Figura 4, donde se puede apreciar que un incremento en el tamaño del lecho, conduce a un mayor tiempo de contacto entre el $A B$ y la $T M$, de tal forma, que una mayor cantidad de $A B$ es retenido por el adsorbente. Lo anterior, permite un mejor aprovechamiento en la zona de transferencia de masa, retardando el tiempo de saturación de la columna y posibilitando de esta manera, el tratamiento de grandes volúmenes de efluentes contaminados. 


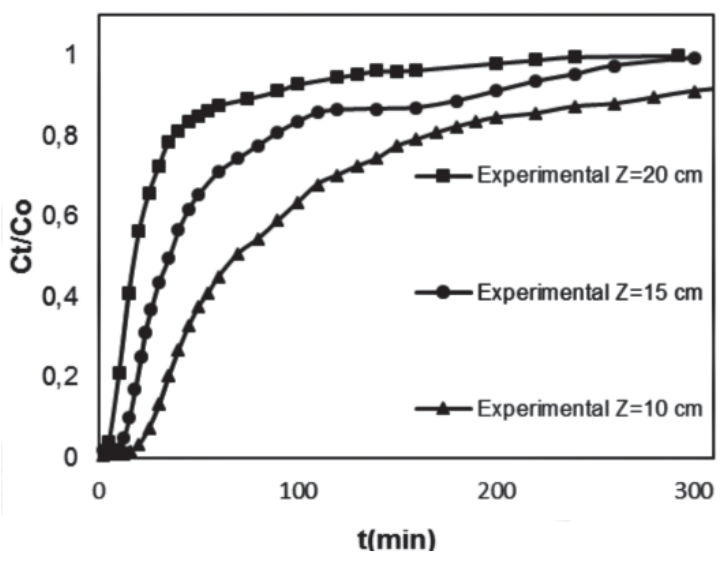

Figura 4. Curvas de ruptura. Análisis del efecto de la altura del lecho en la adsorción de $A B-T M$ $\left(C_{0}=100 \mathrm{mg} \mathrm{L}^{-1}, \mathrm{~V}=20 \mathrm{~mL} \mathrm{~min} \mathrm{~m}^{-1}, \mathrm{pH}=2.0\right.$ )

\section{Modelo de Adams-Bohart para la variable altura de lecho}

El modelo de Adams-Bohart fue aplicado a los datos experimentales para describir la parte inicial de las curvas de ruptura, (Figura 5). Los parámetros característicos, como la máxima capacidad de adsorción $\left(\mathrm{N}_{0}\right)$, la constante cinética $\left(\mathrm{k}_{\mathrm{AB}}\right)$, el error estadístico (SS) y el coeficiente de correlación $\left(R^{2}\right)$, se muestran en la Tabla 1. Cabe señalar, que el modelo fue aplicado haciendo un ajuste a la curva de ruptura desde su parte inicial, hasta la región de concentración relativa $\mathrm{C}_{\mathrm{t}} / \mathrm{C}_{0}=0.1(10 \%)$.

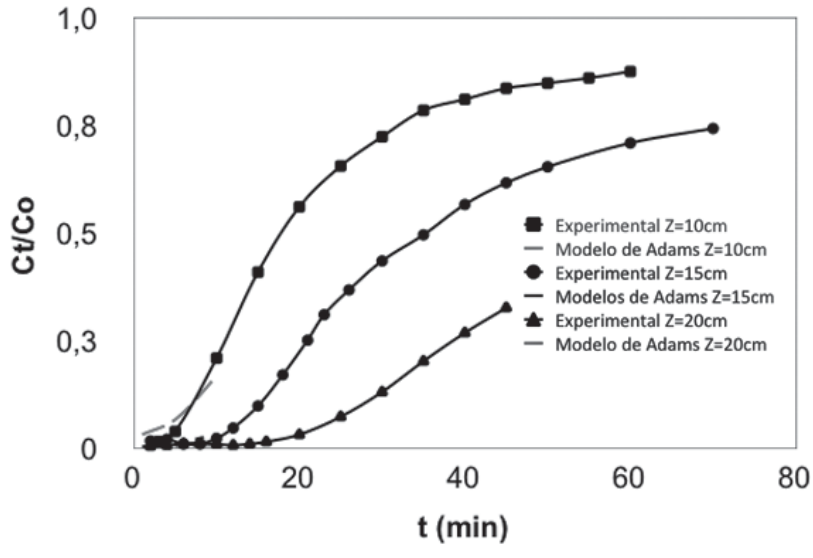

Figura 5. Curvas de ruptura. Modelo de AdamBohart aplicado al efecto de la altura del lecho en la adsorción de $A B-T M\left(C_{0}=100 \mathrm{mg} \mathrm{L}^{-1}\right.$,

$$
V=20 \mathrm{~mL} \mathrm{~min}^{-1}, \mathrm{pH}=2.0 \text { ) }
$$

Los valores de SS señalan un buen ajuste de los datos experimentales a medida que incrementa la altura de columna, $(Z)$, como se aprecia en la Tabla 1 , se encuentran en el intervalo de 0.03 a 0.00 . De igual forma, los valores de $\mathrm{R}^{2}$ confirman esta tendencia, es decir, se alcanza una mejor correlación a mayor altura. Lo anterior, permite sugerir que el modelo de Adams-Bohart es adecuado para predecir el comportamiento inicial de las curvas de ruptura.

Tabla 1. Parámetros del modelo de Adams-Bohart en la remoción de AB usando regresión no lineal

\begin{tabular}{lcccccc}
\hline $\mathbf{C}_{0}\left(\mathrm{mg} \mathrm{L}^{-1}\right)$ & $\mathbf{V}\left(\mathrm{mL} \mathrm{min}^{-1}\right)$ & $\mathbf{Z}(\mathbf{c m})$ & $\mathrm{K}_{\mathrm{A}}\left(\mathrm{Lmg}^{-1} \mathrm{~min}^{-1}\right)$ & $\mathrm{N}_{\mathbf{0}}\left(\mathrm{mgL}^{-1}\right)$ & $\mathbf{R}^{2}$ & $\mathrm{SS}$ \\
100 & 20 & 10 & 0.001761 & 772.91 & 0.96 & 0.03 \\
100 & 20 & 15 & 0.002124 & 654.52 & 0.98 & 0.01 \\
100 & 20 & 20 & 0.001277 & 889.31 & 0.99 & 0.00 \\
\hline
\end{tabular}

\section{Modelo de Thomas para la variable altura de lecho}

Se aplicó el modelo de Thomas, mostrado en la Figura 6, para determinar la constante de velocidad $\left(\mathrm{K}_{\mathrm{TH}}\right)$ y la máxima capacidad de adsorción $\mathrm{q}_{0}\left(\mathrm{mg} \mathrm{g}^{-1}\right)$, a partir de una regresión no lineal a través de la ecuación 1, las cuales junto con el SS y el $R^{2}$ se listan en la Tabla 2. 


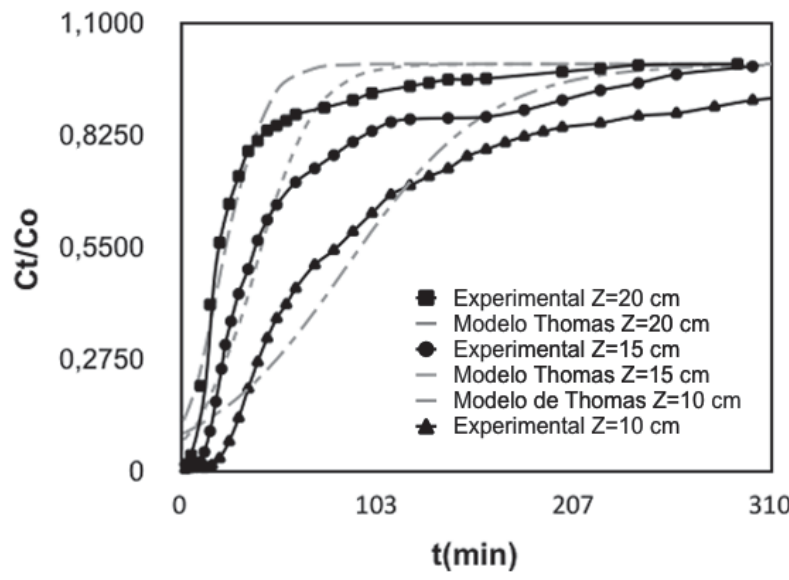

Figura 6. Curvas de ruptura. Modelo de Thomas aplicado al efecto de la altura del lecho en la adsorción de $A B-T M\left(C_{0}=100 \mathrm{mg} \mathrm{L}^{-1}\right.$, $V=20 \mathrm{~mL} \mathrm{~min}^{-1}, \mathrm{pH}=2.0$ )
Como se aprecia en la Tabla 2, a medida que aumenta la altura del lecho, la constante de velocidad disminuye mientras que la capacidad de adsorción incrementa, es decir, se puede alcanzar una mayor remoción del colorante $A B$, debido a que la saturación de la columna requiere un período de tiempo mayor. Con respecto a los valores de SS, ellos se encuentran entre 0.06 y 0.08 , correspondiendo a una buena correlación. Por su parte, el $R^{2}$ varía entre 0.93 y 0.94 , señalando un ajuste aceptable, teniendo en cuenta que se utilizaron 30 puntos experimentales para el ajuste de cada curva.

Tabla 2. Parámetros del modelo de Thomas a diferentes condiciones usando el análisis de regresión no lineal

\begin{tabular}{lcccccc}
\hline $\mathbf{C}_{0}\left(\mathrm{mgl}^{-1}\right)$ & $\mathbf{V}\left(\mathrm{mL} \mathrm{min}^{-1}\right)$ & $\mathbf{Z}(\mathrm{cm})$ & $\mathrm{K}_{\mathrm{TH}}\left(\mathrm{mL} \mathrm{min}^{-1} \mathrm{mg}^{-1}\right)$ & $\mathbf{q}_{\mathbf{0}}\left(\mathrm{mg} \mathrm{g}^{-1}\right)$ & $\mathbf{R}^{2}$ & $\mathrm{SS}$ \\
\hline 100 & 20 & 10 & 0.90 & 5.17 & 0.93 & 0.06 \\
100 & 20 & 15 & 0.66 & 5.46 & 0.94 & 0.08 \\
100 & 20 & 20 & 0.25 & 5.58 & 0.93 & 0.08 \\
\hline
\end{tabular}

\section{Modelo de Yoon - Nelson para la variable altura de lecho}

La constante de velocidad $\mathrm{K}_{\mathrm{YN}}$ y el tiempo requerido para alcanzar el $50 \%$ de saturación de $A B$ en la curva de ruptura, $\tau$, se calcularon a partir del modelo de Yoon-Nelson, mostrado en la Figura 7. Dichos valores se listan en la Tabla 3. Se puede apreciar que al incrementar el tamaño del lecho, los valores de $\tau$ incrementan, mientras que los valores de $\mathrm{K}_{\mathrm{YN}}$ disminuyen. Con relación a los valores de SS y $\mathrm{R}^{2}$, ellos pueden considerarse como aceptables, no obstante, dado que los tiempos experimentales difieren considerablemente de los predichos, el modelo de Yoon-Nelson no es apropiado para predecir el tiempo requerido a una saturación de terminada (Ct/Co).

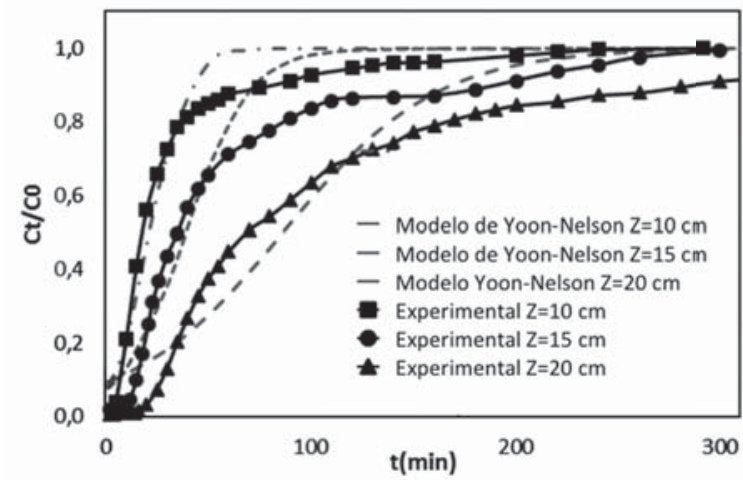

Figura 7. Curvas de ruptura. Modelo de YoonNelson aplicado al efecto de la altura del lecho en la adsorción de $A B-T M\left(C_{0}=100 \mathrm{mg} \mathrm{L}^{-1}\right.$,

$$
V=20 \mathrm{~mL} \mathrm{~min}^{-1}, \mathrm{pH}=2.0 \text { ) }
$$


Tabla 3. Parámetros del modelo de Yoon- Nelson en la remoción de AB usando regresión no lineal

\begin{tabular}{lccccccc}
\hline $\mathbf{C}_{0}\left(\mathrm{mg} \mathrm{L}^{-1}\right)$ & $\mathbf{V}\left(\mathrm{mL} \mathrm{min}^{-1}\right)$ & $\mathbf{Z}(\mathbf{c m})$ & $\mathbf{K}_{\mathrm{YN}}\left(\mathbf{m i n}^{-1}\right)$ & $\tau(\mathrm{min})$ & $\mathbf{R}^{2}$ & $\mathrm{SS}$ & $\tau_{\varepsilon}(\mathrm{min})$ \\
100 & 20 & 10 & 0.13 & 21.93 & 0.96 & 0.07 & 15 \\
100 & 20 & 15 & 0.07 & 38.84 & 0.95 & 0.08 & 35 \\
100 & 20 & 20 & 0.03 & 86.32 & 0.93 & 0.08 & 70 \\
\hline
\end{tabular}

\section{Modelo BDST para la variable altura del lecho}

El modelo BDST se basó principalmente en la medida de la capacidad del lecho empacado a diferentes valores de altura, a una velocidad de flujo y concentración inicial constante e ignorando la resistencia a la transferencia de masa intraparticular y la resistencia de película externa.
Las líneas de $t$ vs $Z$ a valores de $\mathrm{Ct} / \mathrm{Co}$ de $0.2,0.4$ y 0.6 se muestran en la Figura 8 . Las constantes del modelo de acuerdo a la pendiente e intercepto de la línea se listan en la Tabla 4. La capacidad de adsorción del lecho por unidad de volumen, $\mathrm{N}_{\mathrm{o}}$, fue calculada de la pendiente de cada línea y la constante de velocidad, $\mathrm{K}_{\mathrm{a}}$, del intercepto de las misma.

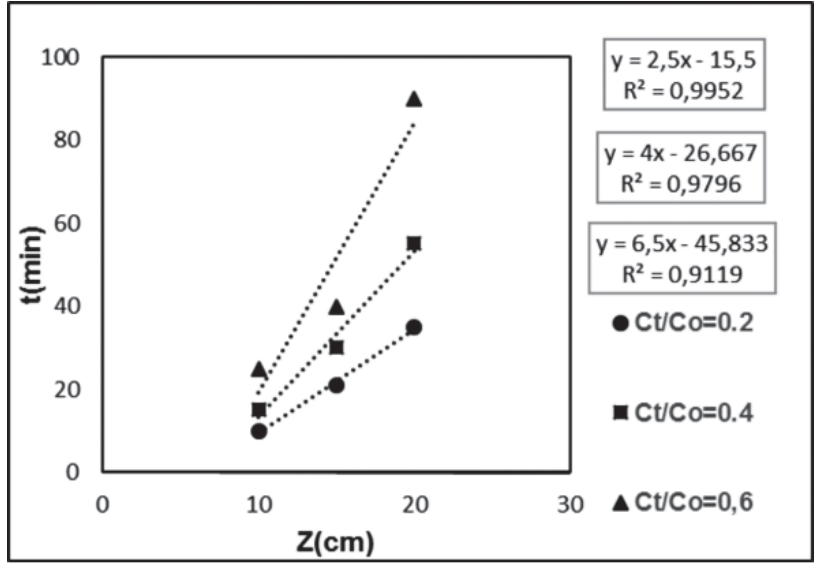

Figura 8. Curvas de ruptura. Análisis del efecto de la altura del lecho en la adsorción de $A B-T M\left(C_{0}=100 \mathrm{mg} \mathrm{L}^{-1}, V=20 \mathrm{~mL} \mathrm{~min}^{-1}, \mathrm{pH}=2.0\right)$

Se observa que al incrementar los valores de Ct/ Co, la constante de velocidad decrece, mientras que la capacidad del lecho por unidad de volumen aumenta. Esto permite establecer que la variable altura es fundamental en la evaluación del proce- so de adsorción como preámbulo a su escalado, usando diferentes velocidades de flujo y concentraciones iniciales de colorante. Finalmente, los valores de $\mathrm{R}^{2}$ señalan la validez de este modelo para representar el sistema.

Tabla 4. Constantes del modelo BDST para la adsorción de AB-TM $\left(C_{0}=100 \mathrm{mg} \mathrm{L}^{-1}, \mathrm{~V}=20 \mathrm{~mL} \mathrm{~min} \mathrm{~m}^{-1}, \mathrm{pH}=2.0\right)$

\begin{tabular}{cccccc}
\hline $\mathbf{C t} / \mathrm{Co}$ & $\mathbf{a}\left(\mathbf{m i n ~ c m}^{-1}\right)$ & $\mathbf{b}(\mathrm{min})$ & $\mathbf{K}_{\mathrm{a}}\left(\mathrm{Img}^{-1} \mathbf{m i n}^{-1}\right)$ & $\mathbf{N}_{\mathrm{o}}\left(\mathrm{mgL}^{-1}\right)$ & $\mathbf{R}^{2}$ \\
0,2 & 2,5 & 15,500 & 0.00086952 & 1362.89 & 0.99 \\
0,4 & 4.0 & 26,667 & 0.00014782 & 2180.63 & 0.98 \\
0,6 & 6,5 & 45,833 & 0.00000881 & 3543.53 & 0.92 \\
\hline
\end{tabular}




\section{Conclusiones}

Los resultados obtenidos sugieren que la TM puede catalogarse como un material adecuado para la remoción eficiente del colorante aniónico $A B$ mediante lechos empacados. El proceso de adsorción en continuo se ve favorecido a pH ácido, $\mathrm{pH}=2.0$, tamaño de partícula entre $0.3<\mathrm{x}<0.5$ $\mathrm{mm}$ y una mayor altura, en este caso particular, $Z=20 \mathrm{~cm}$, dado que estas condiciones incrementan el tiempo requerido para alcanzar la saturación de la columna, permitiendo que mayor cantidad del colorante sea retenida en la columna empacada.

Con respecto al efecto de la solución de $\mathrm{NaCl}$, se encontró que su presencia reduce la eficiencia en la adsorción de $\mathrm{AB}$ bajo sistema continuo como resultado del efecto competitivo entre los iones del colorante y los de la solución salina. En busca de una modelación más ajustada a las condiciones reales de los efluentes industriales, este efecto fue considerado a lo largo de todo el estudio realizado.

Los resultados del comportamiento del proceso muestran que el modelo de Thomas presentó el mejor ajuste para la descripción de las curvas de ruptura a las condiciones experimentales con un $\mathrm{R}^{2}=0.93-0.94$. Por su parte, el modelo de AdamsBohart ofreció un buen ajuste para el comportamiento dinámico limitado a la parte inicial del proceso con un $\mathrm{R}^{2}=0.96-0.99$. Igualmente, el modelo

\section{Literatura citada}

1. Ascu, Z. \& Isoglu, I. (2006). Use of agricultural waste sugar beet pulp for the removal of Gemazol turquoise blue-G reactive dye from aqueous solution. Journal of Hazardous Materials, B137, 418-430.

2. Auxilio, A., Andrews, P., Junk, P., Spiccia, L., Neumann, D., Raverty, D. \& Vanderhoek, N. (2007). Adsorption and intercalation of Acid Blue 9 on Mg-Al layered double hydroxides of variable metal composition. Polyhedron, 26, 3479-3490.

3. Buasri, A., Chaiyut, N., Tapang, K., Jaroensin, S. \& Panphrom, S. (2012). Equilibrium and Kinetic Studies of Biosorption of $\mathrm{Zn}(\mathrm{II})$ lons from Wastewater Using Modified Corn Cob, APCBEE Procedia, 360-64.
BDST señala una correlación adecuada con los datos experimentales, $\mathrm{R}^{2}=0.92-0.99$, a diferencia de lo observado con el modelo de Yoon-Nelson, donde se encontró que las curvas de ruptura se desvían significativamente de las predichas.

Es valioso mencionar que como proyección inmediata de estos resultados se está llevando a cabo en el Grupo de Investigación un escalado preliminar del proceso con alturas de columna de 80 y $100 \mathrm{~cm}$, con el propósito de delinear un lecho filtrante piloto, cuya evaluación permitirá refinar detalles de su funcionamiento hacia una etapa de mayor escalado.

\section{Agradecimientos}

Los autores expresan agradecimientos a la Universidad Nacional de Colombia - Sede Medellín por la dotación en la infraestructura del Laboratorio de Química Experimental. Por la financiación de esta investigación a COLCIENCIAS mediante el otorgamiento de la Beca - Pasantía de Joven Investigador e innovador año 2012, Virginia Gutierréz de Pineda. Igualmente al Programa Nacional de Semilleros de Investigación, Creación e Innovación de la Universidad Nacional de Colombia, Proyecto Código 18484.
4. Crini, G. (2006). Non-conventional low-cost adsorbents for dye removal: a review. Bioresource Technology, 97 (9), 10611-085.

5. El-Fadel, M., Findikakis, A. \& Leckie, J. (1997). Environmental Impacts of Solid Waste Landfilling. Journal of Environmental Management, 90, 1-25.

6. Gupta, V.K., Mittal, A., Krishnan, L. \& Mittal, J. (2005). Adsorption treatment and recovery of the hazardous dye, Brilliant Blue FCF, over bottom ash and de-oiled soya. Journal of Colloid and Interface Science, 293, 16-26.

7. Gupta, V. K. \& Suhas. (2009). Application of low-cost adsorbents for dye removal a review. Journal of Environmental Management, 90 (8), 2313-2342. 
8. Han, R., Ding, D., Xu, Y., Zou W., Wang, Y., Li, Y. \& Zou, L. (2008). Use of risk husk for the adsorption of congo red from aqueous solution in column mode. Bioresource Technology, 99, 2938-2946.

9. Han, R., Wang, Y., Yu, W., Zou, W., Shi, J. \& Liu, H. (2007). Biosorption of methylene blue from aqueous solution by rice husk in a fixed-bed column. Journal of Hazardous Materials, 141, 713-718.

10. Han, R., Wang, Y., Zhao, X., Wang, Y., Xie, F., Cheng, J. \& Tang, M. (2009). Adsorption of methylene blue by phoenix tree leaf powder in a fixed-bed column: experiments and prediction of breakthrough curves. Desalination, 245, 284-297.

11. Hormaza, A., Figueroa, D. \& Moreno, A. (2012). Evaluación de la remoción de un colorante azo sobre tuza de maíz mediante diseño estadístico. Revista de la Facultad de Ciencias Universidad Nacional de Colombia, 1 (1), 61-71.

12. Khataee, A. \& Khataee, H. (2008). Photooxidative removal of the herbicide Acid Blue 9 in the presence of hydrogen peroxide: modeling of the reaction for evaluation of electrical energy per order (EEO). Journal of environmental Science and Health part B, 43, 562-568.

13. Moreno, A., Hormaza, A. \& Figueroa, D. (2012). Diseño estadístico para la remoción eficiente del colorante rojo 40 sobre tuza de maíz. Producción + Limpia, 7 (2), 9-19.

14. Jaramillo, A., Echavarría A. \& Hormaza, A. (2013). Diseño Box-Behnken para la optimización de la adsorción del colorante azul ácido sobre residuos de flores. Revista Ingeniería y Ciencia, 9 (18), 75-91.
15. Ketelsen, H. \& Meyer-Windel S, (1999). Adsorption of brilliant blue FCF by soils. Geoderma, 90, 131-145.

16. Mittal, A. (2005). Use of hen feathers as potential adsorbent for the removal of a hazardous dye, Brilliant Blue FCF, from wastewater. Journal of Hazardous Materials, B128, 233-239.

17. Namasivayam, C., Prabha, D. \& Kumutha, M. (1998). Removal of direct red and acid brilliant blue by adsorption on to banana pith. Bioresource Technology, 64, 7779.

18. Özcan, A. S., Erdem, B. \& Özcan, A. (2004). Adsorption of Acid Blue 193 from aqueous solutions onto $\mathrm{Na}-$ bentonite and DTMA-bentonite. Journal of Colloid and Interface Science, 280, 44-54.

19. Pardo, C. (2012). Selección de Tecnologías Limpias. Recuperado de http://datateca.unad.edu.co/contenidos/358029/ContenidoLinea/ficha_tcnica.htm

20. Piccin, J. S., Vieira, M. L. G., Dotto, G. L. \& Pinto, L. A. (2009). Adsorption of FD\&C Red No. 40 by chitosan: Isotherms analysis. Journal Food Engineering, 95, 16-20.

21. Rafatullah, M. (2010). Adsorption of methylene blue on low-cost adsorbents: a review. Journal of Hazard Materials, 177 (1), 70-80.

22. Song, J., Zou, W., Bian Y., Su, F. \& Han, R. (2011). Adsorption characteristics of methylene blue by peanut husk in batch and column modes. Desalination, 265, 119-125.

23. Wang, S., Zhu, Z.H., Commes, A., Haghseresht, F. \& Lu, G. Q. (2005). The physical and surface chemical characteristics of activated carbons and the adsorption of methylene blue from wastewater. Journal of Colloid and Interface Science, 284, 440-446. 